\title{
Vibrational Density of States of Thin Films Measured by Inelastic Scattering of Synchrotron Radiation
}

\author{
R. Röhlsberger, ${ }^{1}$ W. Sturhahn, ${ }^{2}$ T. S. Toellner, ${ }^{2}$ K. W. Quast, ${ }^{2}$ E. E. Alp, ${ }^{2}$ \\ A. Bernhard, ${ }^{1}$ J. Metge, ${ }^{3}$ R. Rüffer, ${ }^{3}$ and E. Burkel, ${ }^{1}$ \\ 'Universität Rostock, Fachbereich Physik, August-Bebel-Str. 55, 18055 Rostock, Germany \\ ${ }^{2}$ Advanced Photon Source, Argonne National Laboratory," Argonne, Illinois 60439, USA \\ ${ }^{3}$ European Synchrotron Radiation Facility, BP 220, 38043 Grenoble Cedex, France

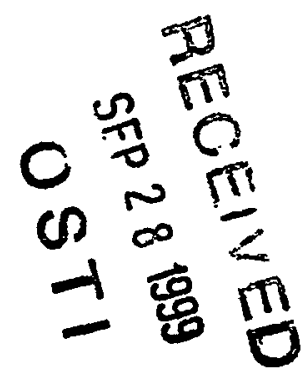

August 1998

The submitted manuscript has been created by
the University of Chicago as Operator of
Argonne National Laboratory ("Argonne") under
Contract No. W-3I-109-ENG-38 with the U.S.
Department of Energy. The U.S. Government
retains for itself, and others acting on its behalf,
a paid-up, nonexclusive, irrevocable worldwide
license in said article to reproduce, prepare
derivative works, distribute copies to the public,
and perform publicly and display publicly, by
or on behalf of the Government.

or on behalf of the Government.

Presented at PHONONS 98, Ninth Intl. Conf. on Phonon Scattering in Condensed Matter, Lancaster, UK, July 26-31, 1998; to be published in the Proceedings.

\footnotetext{
*This work is supported by the U.S. Department of Energy, Basic Energy Sciences-Materials Sciences, under contract \#W-31-109-ENG-38.
} 


\section{DISCLAIMER}

This report was prepared as an account of work sponsored by an agency of the United States Government. Neither the United States Government nor any agency thereof, nor any of their employees, make any warranty, express or implied, or assumes any legal liability or responsibility for the accuracy, completeness, or usefulness of any information, apparatus, product, or process disclosed, or represents that its use would not infringe privately owned rights. Reference herein to any specific commercial product, process, or service by trade name, trademark, manufacturer, or otherwise does not necessarily constitute or imply its endorsement, recommendation, or favoring by the United States Government or any agency thereof. The views and opinions of authors expressed herein do not necessarily state or reflect those of the United States Government or any agency thereof. 


\section{DISCLAIMER}

Portions of this document may be illegible in electronic image products. Images are produced from the best available original document. 


\title{
Vibrational density of states of thin films measured by inelastic scattering of synchrotron radiation
}

\author{
R. Röhlsberger ${ }^{2}$, W. Sturhahn ${ }^{2}$, T. S. Toellner ${ }^{2}$, K.W. Quast ${ }^{2}$, E. E. Alp ${ }^{2}$, \\ A. Bernhard ${ }^{1}$, J. Metge ${ }^{3}$, R. Rüffer ${ }^{3}$, and E. Burkel ${ }^{1}$ \\ ' Universität Rostock, Fachbereich Physik, August-Bebel-Str. 55, 18055 Rostock, Germany \\ ${ }^{2}$ Advanced Photon Source, Argonne National Laboratory, Argonne, IL 60439, USA \\ ${ }^{3}$ European Synchrotron Radiation Facility, BP 220, 38043 Grenoble Cedex, France
}

(July 22, 1998)

\begin{abstract}
Vibrational spectra of thin films were measured by inelastic nuclear resonant scattering of synchrotron radiation in grazing incidence geometry. A strong enhancement of the inelastic signal was obtained by designing the layer system as $\mathrm{x}$-ray waveguide and coupling the incident beam into a guided mode. This effect opens the possibility to study vibrational excitations in thin films that were so far impossible to obtain due to flux limitations.
\end{abstract}

Keywords: Thin films, density of states, synchrotron radiation, Mössbauer effect

Inelastic $\mathrm{x}$-ray scattering studies with meV-energy resolution became feasible in recent years, particularly due to third-generation, undulator-based synchrotron radiation sources [1,2]. A different approach has been introduced to measure phonon spectra via inelastic nuclear resonant scattering from samples containing Mössbauer isotopes like ${ }^{57} \mathrm{Fe}[3-5]$. This method relies on detection of time-delayed fluorescence photons emitted by decaying nuclei in the sample that were excited by synchrotron radiation pulses. If the energy of the radiation is off resonance, resonant excitation of Mössbauer nuclei can still be accomplished by energy exchange with excitations in the sample, e.g. through creation and annihilation of phonons. Therefore, the yield of nuclear decay products like conversion electrons or subsequent $\mathrm{K}$-fluorescence photons gives a measure of the partial phonon density of states in the sample [4]. Due to the outstanding brightness of the undulator radiation, very small sample volumes are sufficient to measure phonon spectra with good statistical quality in short time. In this paper it will be shown how interference effects in grazing incidence geometry can be employed to obtain the vibrational density of states of thin films even for highly absorbing layer materials. The method relies on standing wave formation above total reflecting surfaces and interfaces that may lead to several times the incident intensity in certain regions of the film. This method becomes particularly effective in the case that the film under investigation is coated on a substrate of higher electron density. Between both critical angles of the substrate and the layer material, incident and reflected waves superpose to form a standing wave within the layer [6]. Since the energy transport takes place parallel to the layer boundaries, such layer systems can be regarded as $\mathrm{x}$-ray waveguides [7]. In general, every signal that is derived from the radiation inside the layer can be increased by designing the layer system as an $\mathrm{x}$-ray waveguide and coupling the incident beam into a guided mode by proper adjustment of the angle of inci- dence. Therefore the measurement of phonon spectra of thin films via detection of delayed fluorescence photons will benefit from the interference effect described above.

For a given phonon energy transfer $E$, the flux of delayed $\mathrm{K}$-fluorescence photons emitted from the film into the full solid angle is given by [4]:

$$
J(E)=I_{0} \rho \sigma \frac{\eta_{K} \alpha_{K}}{1+\alpha} \frac{\pi}{2} \Gamma_{0} S(E) \int_{0}^{d} I(z) d z
$$

where $I(z)$ is the depth dependence of the x-ray intensity inside the film. $I_{0}$ is the incident flux, $\rho$ is the area density of the nuclei as seen from the direction of the incident beam, $\sigma$ the nuclear resonant cross section, $\eta_{K}$ the fluorescence yield. $\alpha$ and $\alpha_{K}$ are the total and partial internal conversion coefficients, respectively, and $\Gamma_{0}$ $=4.66 \mathrm{neV}$ is the level width of the $14.4 \mathrm{keV}$ resonance of ${ }^{57} \mathrm{Fe} . S(E)$ is the absorption probability per unit of energy, equivalent to the momentum-integrated dynamic structure factor of the $\mathrm{Fe}$ atoms in the sample.

The experiments were carried out at the undulator beamlines 3ID [8] and ID18 [9] of the Advanced Photon Source (APS), Argonne National Lab., USA and the European Synchrotron Radiation Facility (ESRF), Grenoble, France, respectively. The experimental setup is shown schematically in fig.1a. The incident radiation was monochromatized to an energy bandwidth of $4-6$ $\mathrm{meV}$ by a high-resolution monochromator (HRM) [10]. Delayed fluorescence photons were detected by placing the detector (avalanche photodiode, APD) right above the film so that a solid angle as large as possible was covered.

Fig. 1b displays the experimental results for a $19.5 \mathrm{~nm}$ $\mathrm{Fe}_{2} \mathrm{Cr}_{2} \mathrm{Ni}$ film on $10 \mathrm{~nm} \mathrm{Pd}$ coated on a superpolished substrate. To demonstrate the intensity enhancement of the inelastic signal, the $\mathrm{x}$-ray energy was tuned $20 \mathrm{meV}$ above the resonance, and the angular dependence of the delayed $6.4 \mathrm{keV}$ fluorescence yield was recorded, shown 
in fig. Ib. The curve shows a pronounced maximum at an angle of $4.1 \mathrm{mrad}$, where an intense standing wave inside the film forms. At this angular position a phonon spectrum was recorded within a data acquisition time of just one hour, shown in the left inset of fig. $1 \mathrm{~b}$.
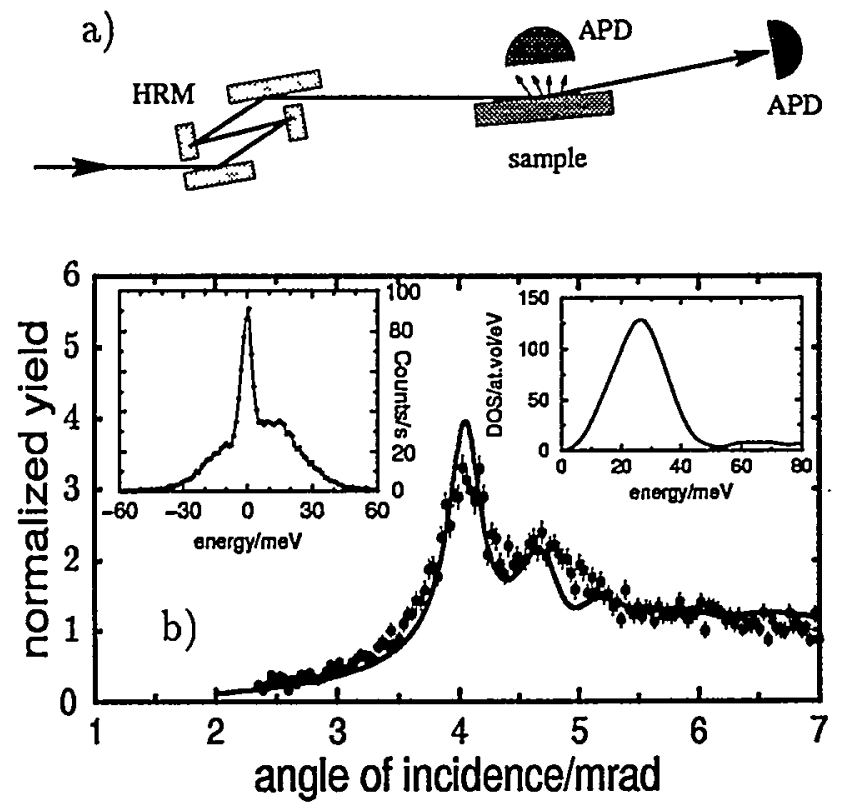

FIG. 1. a) Experimental setup, HRM: High-resolution monochromator (5.6 meV), APD: Avalanche photodiode. b) Angular dependence of the delayed nuclear fluorescence yield from $19 \mathrm{~nm}^{57} \mathrm{Fe}_{2} \mathrm{Cr}_{2} \mathrm{Ni}$ on $\mathrm{Pd}$, normalized to the value at normal incidence. The $x$-ray energy was $20 \mathrm{meV}$ above the 14.4 $\mathrm{keV}$ resonance. The left inset shows the phonon spectrum recorded at the peak yield at $4.2 \mathrm{mrad}$. The right inset shows the DOS derived form the phonon spectrum. (Experiment performed at the APS, Argonne Natl. Lab.)

From this measurement the phonon DOS was extracted as explained in detail in [4], shown in the right inset in fig.1b. The DOS is a single broad peak without fine structure. This can be qualitatively explained by a distribution of bond angles and bond lengths in the amorphous state that leads to a broad distribution of phonon energies instead of well defined modes at fixed frequencies. Moreover, lifetime effects due to reduced dimensionality in thin films lead to phonon damping and broadening of phonon modes, as observed recently in $\mathrm{Fe}$ nanocrystals [11] and Fe thin films [12].

The intensity enhancement due to thin film interference can be even more efficiently used, if the investigated film is sandwiched between two total reflecting layers. This has been done in case of a $13 \mathrm{~nm}$ thick layer of sputtered $\mathrm{FeBO}_{3}$, where the fraction of ${ }^{57} \mathrm{Fe}$ in $\mathrm{Fe}$ was only $2 \%$ (natural abundance). The measurement was possible due to a 10-fold enhancement of the $\mathrm{x}$-ray intensity in a $\mathrm{Pd} / \mathrm{FeBO}_{3} / \mathrm{Pd}$ waveguide structure. The amount of ${ }^{57} \mathrm{Fe}$ in that sample was equivalent to one monolayer. The phonon spectrum is shown in fig.2, recorded at the ESRF with an energy resolution of $4.4 \mathrm{meV}$. Here the data acquisition time was about 6 hours. The phonon spectrum already shows that the phonon energies in this sample are considerably lower than in the case of the $\mathrm{Fe}_{2} \mathrm{Cr}_{2} \mathrm{Ni}$ film.

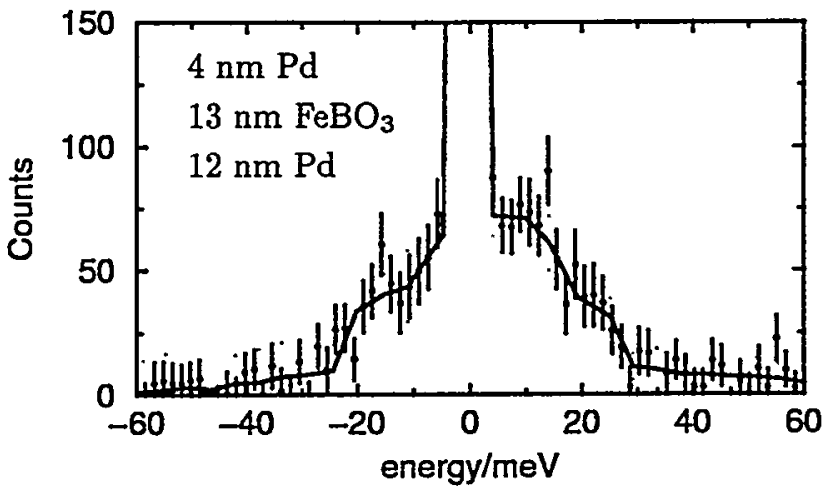

FIG. 2. ${ }^{57} \mathrm{Fe}$ phonon spectrum of $13 \mathrm{~nm} \mathrm{FeBO}_{3}$ in a $\mathrm{Pd} / \mathrm{FeBO}_{3} / \mathrm{Pd}$ waveguide structure. The energy resolution was $4.4 \mathrm{meV}$. The solid line is a guide to the eye. (Experiment performed at the ESRF, Grenoble)

In conclusion, the high sensitivity of the method together with the inherent isotope specifity allows to investigate local vibrational properties of low dimensional systems, perhaps down to the atomic level, e.g. by selective enrichment of the resonant isotope. The experiments demonstrate a great potential for inelastic spectroscopy of thin films at 3 rd generation synchrotron radiation sources.

We gratefully acknowledge the continuous support of E. Gerdau and the late M. Harsdorff during preparation of the films at the University of Hamburg. This work was supported by the U.S. Department of Energy, BES Materials Science, under Contract No. W-31-109-ENG38 and by the German BMBF under contract no. 05 643HRA 5

[1] E. Burkel, Inelastic Scattering of X-Rays with Very High Energy Resolution (Springer-Verlag, New York, 1991)

[2] F. Sette et al., Phys. Rev. Lett. 77, 83 (1996)

[3] M. Seto et al., Phys. Rev. Lett. 74, 3828 (1995)

[4] W. Sturhahn et al., Phys. Rev. Lett. 74, 3832 (1995)

[5] A. I. Chumakov et al., Europhys. Lett. 30, 427 (1995)

[6] A. Krol et al., Phys. Rev. B 38, 8579 (1998)

[7] Y. P. Feng et al., Phys. Rev. Lett. 71, 537 (1993)

[8] E. E. Alp et al., Hyp. Int. 90, 323 (1994)

[9] R. Rüffer et al., Hyp. Int. 97/98, 589 (1996) 
[10] T. M. Mooney et al., Nucl. Instrum. Methods A 347, 348 (1994)

[11] B. Fultz et al., Phys. Rev. Lett. 79, 937 (1997)

[12] R. Röhlsberger et al., to be published 